\title{
Glycogen Metabolism in Vibrio vulnificus Affected by malP and malQ
}

\author{
Ah-Reum Han ${ }^{1}$, Yeon-Ju Lee ${ }^{1}$, Tianshi Wang ${ }^{1 \#}$, and Jung-Wan Kim ${ }^{1,2 *}$ \\ ${ }^{1}$ Department of Life Sciences, Graduate School of Incheon National University, ${ }^{2}$ Division of Bioengineering, Incheon National University, \\ Incheon 22012, Republic of Korea \\ ${ }^{\#}$ current address: Department of Biochemistry and Molecular Cell Biology Shanghai Key Laboratory for Tumour Microenvironment and \\ Inflammation, Shanghai Jiao Tong University School of Medicine, Shanghai 200025, China
}

Received: January 8, 2018 / Revised: March 6, 2018 / Accepted: March 6, 2018

\begin{abstract}
Vibrio vulnificus needs various responsive mechanisms to survive and transmit successfully in alternative niches of human and marine environments, and to ensure the acquisition of steady energy supply to facilitate such unique life style. The bacterium had genetic constitution very different from that of Escherichia coli regarding metabolism of glycogen, a major energy reserve. $V$. vulnificus accumulated more glycogen than other bacteria and at various levels according to culture medium and carbon source supplied in excess. Glycogen was accumulated to the highest level in Luria-Bertani $(3.08 \mathrm{mg} / \mathrm{mg}$ protein) and heart infusion $(4.30 \mathrm{mg} / \mathrm{mg}$ protein) complex media supplemented with $1 \%(\mathrm{w} / \mathrm{v})$ maltodextrin at $3 \mathrm{~h}$ into the stationary phase. Regarding effect of carbon source, more glycogen was accumulated when maltodextrin (2.34 $\mathrm{mg} / \mathrm{mg}$ protein) was added than when glucose or maltose (0.78-1.14 $\mathrm{mg} / \mathrm{mg}$ protein) was added as an excessive carbon source to M9 minimal medium, suggesting that maltodextrin metabolism might affect glycogen metabolism very closely. These results were supported by the analysis using the malP (encoding a maltodextrin phosphorylase) and malQ (encoding a 4- $\alpha$-glucanotransferase) mutants, which accumulated much less glycogen than wild type when either glucose or maltodextrin was supplied as an excessive carbon source, but at different levels (3.1-80.3\% of wild type glycogen). Therefore, multiple pathways for glycogen metabolism were likely to function in $\mathrm{V}$. vulnificus and that responding to maltodextrin might be more efficient in synthesizing glycogen. All of the glycogen samples from $3 \mathrm{~V}$. vulnificus strains under various conditions showed a narrow side chain length distribution with short chains (G4-G6) as major ones. Not only the comparatively large accumulation volume but also the structure of glycogen in $\mathrm{V}$. vulnificus, compared to other bacteria, may explain durability of the bacterium in external environment.
\end{abstract}

Keywords: Vibrio vulnificus, glycogen metabolism, maltodextrin metabolism, ma/P, malQ, side chain length distribution

\section{Introduction}

Vibrio vulnificus is a gram negative, rod shaped marine bacterium that is motile with flagella and known as an opportunistic and highly invasive human pathogen. The bacterium frequently causes gastroenteritis and life-threatening septicemia via intake of raw or

\section{*Corresponding author}

Tel: +82-32-835-8244, Fax: +82-32-835-8680

E-mail: kjw5864@inu.ac.kr

(c) 2018, The Korean Society for Microbiology and Biotechnology under-cooked seafoods, and also via wound infection by seawater [1, 2]. Septicemia causes various symptoms of fever, chills, nausea, hypotensive septic shock, tragic secondary lesions on extremities of patients [3], and leads immunocompromised patients or individuals with basal liver disease to high mortality over 50\% [4].

$V$. vulnificus can undergo two life cycles, one in the host and the other in aquatic environment via multistage process of invading and escaping the host. First, it needs to be ingested at a dose high enough to overcome host immune defense, secondly express virulent factors, 
and finally control exit from the host to facilitate transmission to the environment [5, 6]. A previous study on $V$. cholerae reported that the pathogen survived in nutrient rich human hosts and nutrient poor aquatic environments successfully [7]. V. cholerae might extend opportunity for survival by forming biofilm and utilizing chitin in aquatic environments, while it would colonize on the surface of small intestine and secrete cholerae toxin (CT) to cause diarrhea for exit from the host $[7,8]$. V. vulnificus as well as $V$. cholerae would encounter various challenges such as shifts in osmolality, $\mathrm{pH}$, and temperature as well as limitation of crucial nutrients such as carbon, nitrogen, or phosphate source due to dramatic changes during the life cycles [5, 7]. However, cellular or genetic changes leading $V$. vulnificus to overcome transition between the human host and aquatic environment successfully have not been elucidated clearly yet, especially regarding the role of glycogen metabolism in that matter.

Glycogen, one of the most common energy sources for various organisms, is consisted of glucose residues linked mainly by $\alpha$-1,4-glucosidic linkages (90\%) and by $\alpha$-1,6-glucosidic linkages (10\%) at every branch points [9]. Bacteria accumulate glycogen when limitation of nitrogen or phosphate source affects their growth in the presence of excess carbon source [10]. Studies on glycogen metabolism have revealed that glycogen might have crucial functions in regulating life cycles or virulence in bacteria. Glycogen reservoir in $V$. cholerae was reported to be very important in successful transmission between host and environment, ensuring prolonged survival in the latter [8]. Moreover, bacterial glycogen with shorter average chain length (ACL) had slower breakdown rate and enhanced bacterial durability [11]. Therefore, both amount as well as structure of glycogen in bacteria would be important for the survival of bacteria.

Many genes are involved in bacterial glycogen metabolism and have been investigated most widely for those in Escherichia coli: glgA (glycogen synthase), glgC (ADPglucose pyrophosphorylase), and $g \lg B$ (glycogen branching enzyme) are for glycogen synthesis; $g \lg X$ (glycogen debranching enzyme) and glgP (glycogen phosphorylase) for glycogen degradation [12]. Investigations on the glg$B X C A P$ operon revealed differential regulations on these genes during growth under various conditions for synthesis and utilization of glycogen in the bacterium [13]. Constitution of these genes was variable among bacteria, affecting their capacity of glycogen metabolism and persistence in nature. In $V$. cholerae, induction of the malPQ-glgB operon and the glgX gene was detected in human intestine, while $g \lg X$ was repressed in the bacteria shed via rice-water stool $[8,14]$.

Genes for maltose/maltodextrin metabolism are also involved in glycogen metabolism to form multiple pathways for it in E. coli [15]. Maltose and short maltodextrins entering the cells are hydrolyzed to glucose from the reducing ends by a 4- $\alpha$-glucanotransferase encoded by malQ. MalQ transfers the remaining enzyme bound dextrinyl residue to the non-reducing ends of other maltodextrins, forming longer maltodextrin chains [16]. Then, resulting larger maltodextrins are linked to glycogen molecules by GlgB to form branch points. A maltodextrin phosphorylase encoded by malP removes glucose
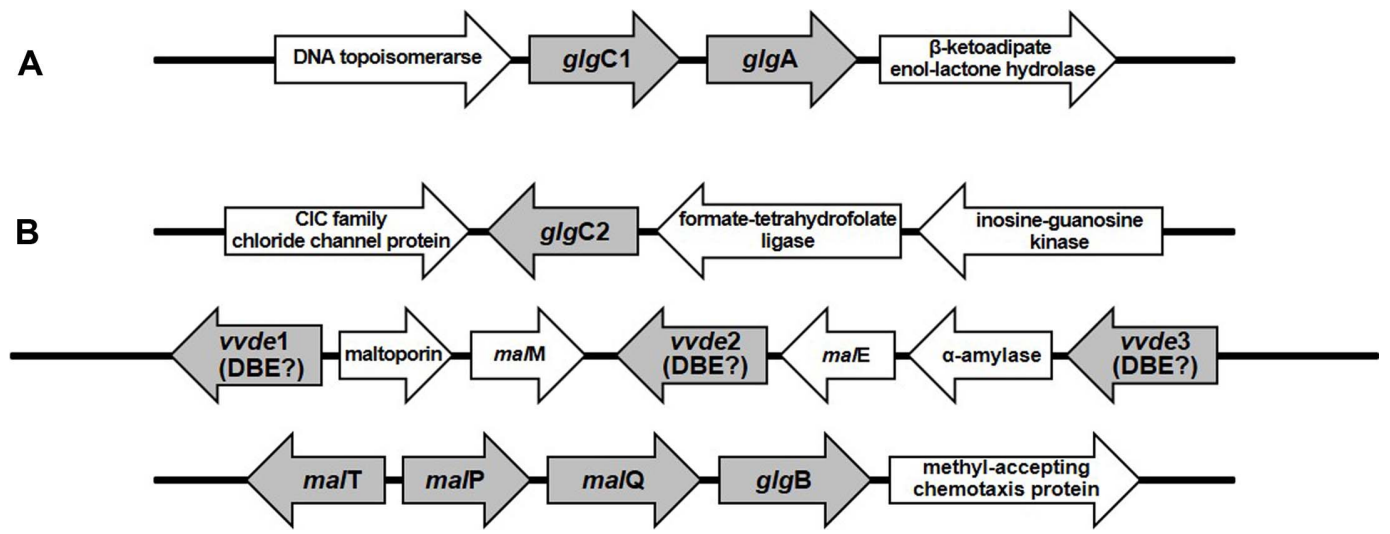

Fig. 1. Clusters of the genes that might be related to glycogen metabolism in V. vulnificus. The genes for glycogen metabolism are shown in shade. Only the $g l g C 1$ and glgA genes are located on chromosome I (A). While the others are scattered over chromosome II (B). DBEs represent genes that are annotated as encoding putative debranching enzymes in the $V$. vulnificus genome. 
from non-reducing ends of maltotetraose and longer maltodextrins and phosphorylate it to form glucose-1-P for the glycolysis pathway. These genes, therefore, make link between glycogen metabolism and glucose/maltose/ maltodextrin metabolisms.

Genetic make-up of these genes in $V$. vulnificus is unique in that the bacterium has two glgC genes, each on chromosome I, II and the malPQ are closely linked to glgB (Fig. 1). Only one phosphorylase, MalP (VVMO6_ 03056), was annotated regarding maltodextrin utilization in the bacterial genome, having 69.1\% (53.9\%) and $62.5 \%$ (44.4\%) homology (identity) to E.coli MalP and GlgP, respectively. V. vulnificus MalQ shared 64.8\% (47.5\%) homology (identity) with that of $E$. coli. MalP and MalQ of $V$. vulnificus were larger than those of $E$. coli by 20 and 32 amino acids, respectively, with more variance at the amino terminus. Previously, malPQ were reported that they were related to metabolism of maltose in $V$. vulnificus [17]. These indicated that $V$. vulnificus might have unique glycogen metabolism pathways or regulatory systems more closely linked to maltodextrin metabolism rather than maltose metabolism, which remained to be elucidated further.

In this study, we report investigation on glycogen metabolism in $V$. vulnificus by analyzing glycogen accumulation in the bacterium under various growth conditions. Also, roles of the malP and malQ genes in glycogen metabolism were examined by using knock-out mutants. Structure of the glycogen molecules synthesized by the bacterium was examined by analyzing side chain length distribution. The results obtained in this study would provide information for understanding the life cycle and monitoring the transmission of this pathogen in nature.

\section{Materials and Methods}

\section{Strains and culture conditions}

Vibrio vulnificus MO6-24/O, a clinical isolate, and its mutants were provided by Prof. SH Choi, Seoul National University (Korea). They were generally cultured at $37^{\circ} \mathrm{C}$ in Luria-Bertani broth [LB; $1 \%(\mathrm{w} / \mathrm{v})$ tryptone, $0.5 \%$ (w/v) yeast extract, and $2 \%(\mathrm{w} / \mathrm{v}) \mathrm{NaCl}$; Difco, USA] with shaking (200 rpm). M9 minimal medium [0.6\% (w/v) $\mathrm{Na}_{2} \mathrm{HPO}_{4}, 0.3 \%$ (w/v) $\mathrm{KH}_{2} \mathrm{PO}_{4}, 2.0 \%$ (w/v) $\mathrm{NaCl}, 0.1 \%$ (w/v) $\mathrm{NH}_{4} \mathrm{Cl}, 0.1 \mathrm{mM} \mathrm{CaCl}, 0.2 \% \mathrm{MgSO}_{4}, 0.5 \%$ (w/v) carbon source]. Heart-infusion broth [HI; 1\% (w/v) beef heart infusion, $1 \%(\mathrm{w} / \mathrm{v})$ tryptose, $2.0 \%(\mathrm{w} / \mathrm{v}) \mathrm{NaCl}]$ as well as LB and M9 minimal medium containing various carbon sources were used for glycogen analysis. For agar plate analysis, $1.5 \%(\mathrm{w} / \mathrm{v})$ agar was added to each medium.

\section{lodine vapor test}

Glycogen accumulated in the bacterium was visualized by the iodine vapor test $[18,19]$. The cells were cultured on LB, HI, or M9 minimal agar plates containing $1 \%(\mathrm{w} / \mathrm{v})$ glucose, maltose, or maltodextrin for $24-48 \mathrm{~h}$ at $37^{\circ} \mathrm{C}$ before they were stained with iodine vapor. The cell patches formed on the plates were stained by placing the plates upside down on a beaker containing potassium iodine solution $\left[2 \% \mathrm{I}_{2}(\mathrm{w} / \mathrm{v}), 0.4 \% \mathrm{KI}(\mathrm{w} / \mathrm{v})\right]$ for $1 \mathrm{~min}$ under a fume hood.

\section{Extraction and quantitation of glycogen}

$V$. vulnificus was cultured in an appropriate medium $(500 \mathrm{ml})$ at $37^{\circ} \mathrm{C}$ with shaking $(200 \mathrm{rpm})$ until the culture reached late exponential growth phase and 1\% (w/v) maltodextrin (DE=10; Daesang Co., Korea), glucose, or maltose was added as a carbon source to enhance glycogen accumulation. Then, an aliquot of each culture (50 ml) was taken at every $2 \mathrm{~h}$ from the time point of adding maltodextrin. The cells were harvested by centrifugation at $6,000 \times g$ (Supra22K, Hanil, Korea) for $10 \mathrm{~min}$ and washed with $5 \mathrm{ml}$ of ice-cold water. Each cell pellet was resuspended in $2.5 \mathrm{ml}$ of $50 \mathrm{mM}$ sodium-acetate buffer ( $\mathrm{pH} 4.5$ ) and vortexed. The resuspension was boiled for $10 \mathrm{~min}$ and sonicated on an ice bath (Sonoplus ultrasonic homogenizer HD2070, Bandeln, Germany: output $5 \mathrm{~min} \times 2$ times, $50 \%$ duty). The crude cell extract was centrifuged at $10,000 \times g$ for $20 \mathrm{~min}$ at $25^{\circ} \mathrm{C}$. Glycogen in the supernatant was precipitated by adding 2 volumes of ethanol. Precipitated glycogen was isolated by centrifugation at $10,000 \times \mathrm{g}$ for $20 \mathrm{~min}$ at $4^{\circ} \mathrm{C}$ and the supernatant was removed before air-dry. Dried glycogen was resuspended in $1 \mathrm{ml}$ of sterile distilled water and stored at $-20^{\circ} \mathrm{C}$ until use for further analysis [20].

The amount of glycogen isolated from $V$. vulnificus was determined using the anthron-sulfate method [21]. Fifty microliters of each sample were mixed with $10 \times$ volumes of a solution [84\% (v/v) sulfuric acid containing $0.7 \%(\mathrm{w} / \mathrm{v})$ anthron] and was incubated in an oven set at 
$110^{\circ} \mathrm{C}$ for $10 \mathrm{~min}$. After cooled down to room temperature, absorbance of the reaction was measured at $595 \mathrm{~nm}$ using a spectrophotometer (Ultrospec 2000, Pharmacia Biotec, USA) and the amount of glycogen was determined by extrapolating the OD values to a standard curve prepared using pure glycogen (Sigma, USA).

\section{Side chain length analysis}

To analyze structure of glycogen by determining lengths of branches, glycogen isolated from the bacteria was hydrolyzed using an isoamylase $(0.4 \mathrm{U} / \mathrm{mg}$ of substrate; Hayashibara Biochemical Laboratories Inc., Japan) in $50 \mathrm{mM}$ sodium acetate buffer (pH 4.3) at $60^{\circ} \mathrm{C}$

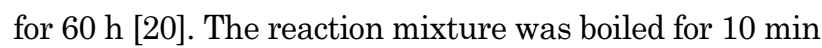
to stop the debranching reaction before centrifuged at $12,000 \times g$ for $10 \mathrm{~min}$ and filtered using a membrane ( $0.45 \mu \mathrm{m}$, Millipore, USA). The pre-treated samples were analyzed by high performance anion exchange chromatography (HPAEC; Dionex DX-500 system, USA) equipped with a pulsed amperometric detector (ED40, Dionex, USA) and a CarboPac PA- 1 column $(4 \times 250 \mathrm{~mm}$, Dionex, USA). Samples or standard markers (G1-G7) were injected and eluted with a gradient of $0-60 \mathrm{mM}$ sodium acetate $(\mathrm{pH} 4.3)$ in $0.15 \mathrm{M} \mathrm{NaOH}$ at a flow rate of $1.0 \mathrm{ml} / \mathrm{min}$ : a linear gradient of $10-30 \%$ for $0-10 \mathrm{~min}$, $30-40 \%$ for $10-16 \mathrm{~min}, 40-50 \%$ for $16-27 \mathrm{~min}, 50-60 \%$ for $22-44 \mathrm{~min}$, and $100 \%$ for $44-60 \%$.

\section{Results and Discussion}

\section{Effect of culture medium and carbon source on glycogen accumulation}

As an effort to detect glycogen accumulation in $V$. vulnificus, the bacterium was cultured on various agar plates (M9 minimal medium, LB, or HI) supplemented with an excessive carbon source (glucose, maltose, or maltodextrin; $1 \%$, w/v) to enhance glycogen synthesis. Cell patches formed on the agar plates were stained by iodine vapor at various degrees according to the amount and the structure of glycogen accumulated in the cells under each growth conditions (Fig. 2). Cells stained to dark brown illustrated more glycogen accumulation with more branches, while those stained to light brown or not stained represented poor or no glycogen accumulation, respectively $[18,19]$. V. vulnificus was stained to the darkest color on the agar plates supplemented with

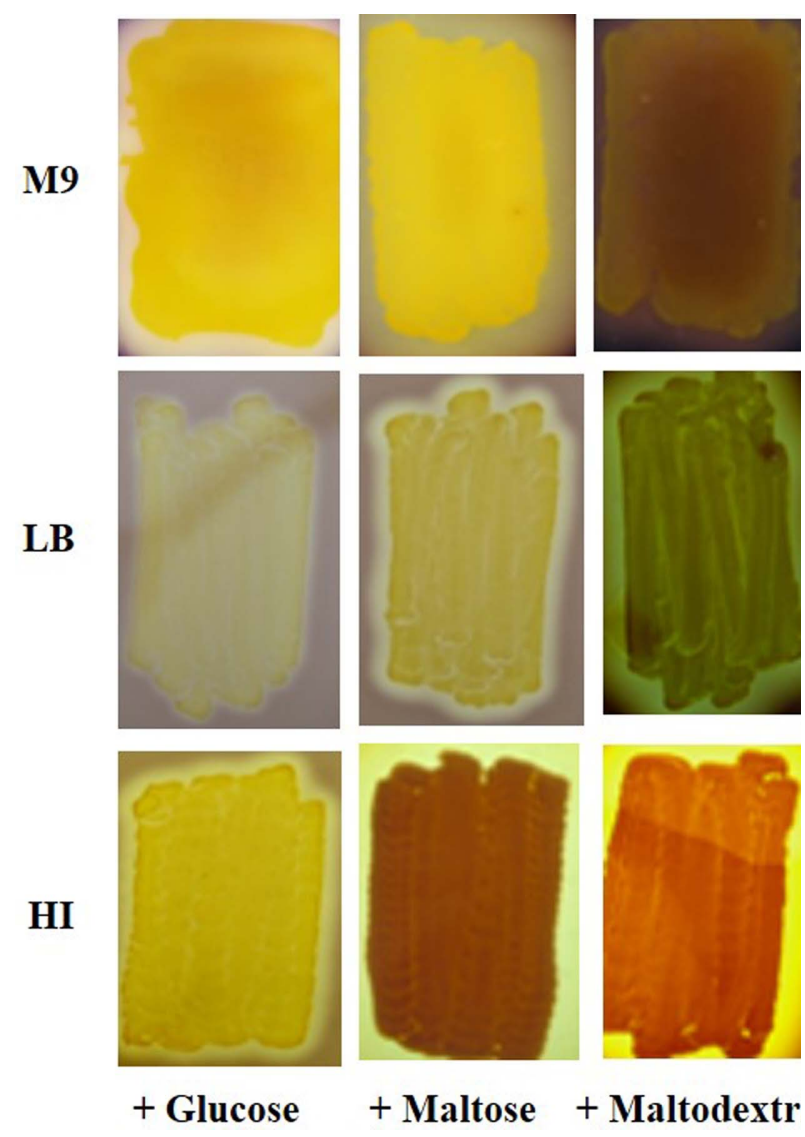

Fig. 2. Agar plate assay of glycogen accumulation using iodine vapor staining. Glycogen accumulated in the cells of $V$. vulnificus was detected by staining the cell patches with iodine vapor. $V$. vulnificus was cultured on various agar plates supplemented with excessive carbon sources for $24-48 \mathrm{~h}$ at $37^{\circ} \mathrm{C}$ before exposed to iodine vapor.

maltodextin and the lightest in the presence of glucose generally, suggesting that each carbon source might affect glycogen metabolism in the bacterium via stimulating certain metabolic pathway(s). This implied that transport and catabolism of maltodextrin in $V$. vulnificus were likely to be very closely linked to glycogen synthesis. Interestingly, among the media used for the agar plate assay, the cells on HI agar plates were stained to the darkest color regardless the type of carbon source supplemented in an excess amount. The cells were stained darker brown and red brown in the presence of maltose and maltodextrin, respectively, implying difference in structures of glycogen molecules formed under the two conditions. This could be due to the difference in the pathways for glycogen synthesis when the two different 
carbon sources were supplied, which remained to be elucidated. It is not clearly understood why HI medium supported accumulation of glycogen of the bacterium better than LB medium either, even though both were complex rich media. This could be due to the difference in the composition of nutrients or in the growth rate of $V$. vulnificus cultured in these media, implying that the metabolism is modulated by various factors in and outside of the bacterium. The results from the iodine vapor staining assay do not simply represent quantitative glycogen accumulation, but combined effect of amount and structural characteristics of the molecule. Therefore, quantitative analyses of glycogen were carried out for better understanding of glycogen metabolism in $V$. vulnificus under various culture conditions.

\section{Accumulation of glycogen during the course of growth in V. vulnificus}

Accumulation of glycogen in the wild type (WT) V. vulnificus strain was monitored more in detail and analyzed quantitatively by time-course assay using various culture media and under various culture conditions. First, glycogen accumulation in $V$. vulnificus WT was monitored using complex media (LB and HI) and maltodextrin as an excess carbon source, which caused the most significant accumulation of glycogen in the agar plate assay. When no additional carbon source was added to the media, the bacteria began to accumulate glycogen during exponential growth phase and the amount was kept almost constant during the rest of the culture period (Fig. 3). However, accumulation of glycogen was boosted upon the addition of excessive amount of maltodextrin $(1 \%, \mathrm{w} / \mathrm{v})$ during the exponential growth phase and was kept increasing for $8 \mathrm{~h}$. The bacterial growth itself was not affected by the excessive carbon source, indicating that other growth factor(s) was limited under the conditions. At each time point analyzed further, more glycogen was accumulated when the cells were cultured in HI medium than in LB medium (Fig. 3). Taking the growth rate of each culture into account, glycogen levels began to increase as the growth rate decreased and reached the highest level at the $3 \mathrm{~h}$ into stationary phase. Continuous increase in glycogen accumulated in the cultures supplied with an excessive carbon source or maintenance of glycogen amount in the cultures without supply of an excessive carbon source indicated that the molecule was not used readily.

The growth rate and cell cycle dependent accumulation or degradation of glycogen have been reported for yeast and Mycobacterium smegmatis [22, 23]. Glycogen is known to be accumulated in E. coli and Aerobacter aerogenes when excessive carbon source is available but other growth factors such as nitrogen and/or phosphate is limited [24, 25]. Also, in V. cholerae, insufficient concentration of nitrogen led bacteria to accumulate more glycogen up to $\sim 15$ folds [8]. V. vulnificus could accumulate more glycogen than E. coli and Bacillus subtilis by about 10 to 20 folds when cultured in LB supplemented
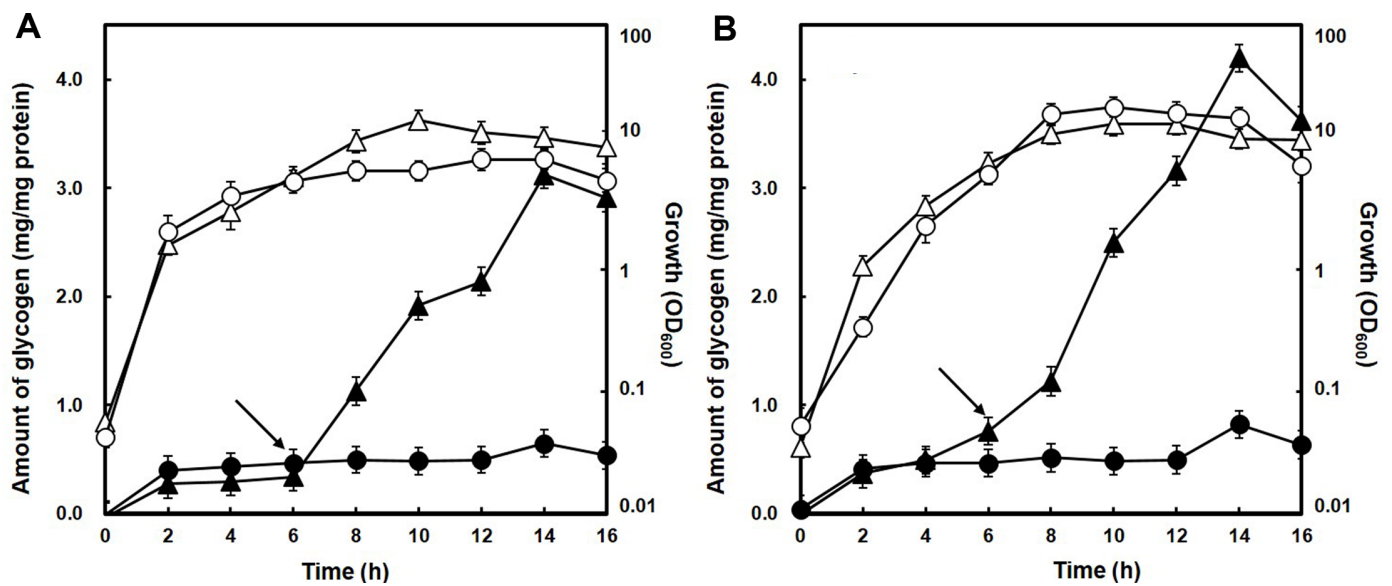

Fig. 3. Time course assay of glycogen accumulation in $\boldsymbol{V}$. vulnificus using complex media. The wild type $V$. vulnificus strain was cultured in LB (A) or HI (B) broth. The graphs marked with open symbols represent growth of $V$. vulnificus; closed symbols, amount of glycogen. The graphs marked with circles represent cultures with no carbon source added; triangles, with $1 \%$ (w/v) maltodextrin at added the $6 \mathrm{~h}$ of cultivation (marked with an arrow). 
with excessive maltodextrin (1\%) during exponential growth phase [20,26]. This could be due to unique genetic make-up of the genes related to carbohydrate and sugar metabolism in $V$. vulnificus, which has to face two significantly different environments to survive.

\section{Effects of carbon source on glycogen accumulation}

Secondly, effects of carbon source on glycogen accumulation in V. vulnificus WT were examined using M9 minimal medium supplemented with $0.5 \%$ (w/v) glucose, maltose, or maltodextrin at the beginning of cultivation and then each carbon source was added in extra (1\%, w/v) at the $6 \mathrm{~h}$ of cultivation (Fig. 4). When glucose or maltose was added as the excessive carbon source, glycogen accumulation of the bacterium was poor. On the other hand, when maltodextrin was added to the culture in excess, glycogen began to be accumulated at the mid-exponential growth phase to the amount much higher than those from the culture with glucose or maltose and reached the highest level at the $14 \mathrm{~h}$ of cultivation (Fig. 4). Therefore, maltodextrin taken up into the $V$. vulnificus cells induced the synthesis of glycogen much more efficiently than glucose or maltose under the conditions investigated, implying that the bacterium might have glycogen metabolism pathways different from those of $E$. coli.

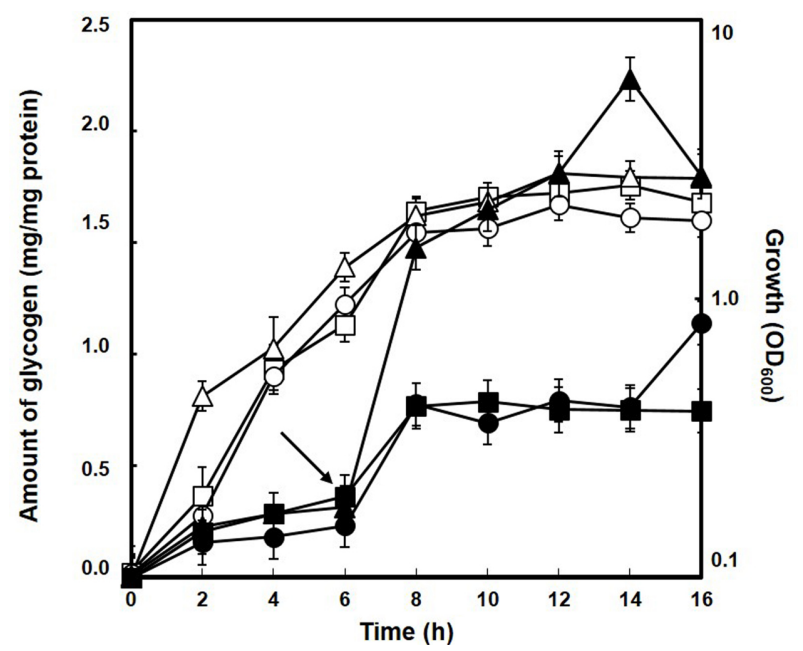

Fig. 4. Time course assay of glycogen accumulation in $\mathbf{V}$. vulnificus using M9 minimal broth. The graphs with open symbols represent growth of $V$. vulnificus; closed symbols, amount of glycogen; circles, with excessive glucose; squares, with excessive maltose; triangles, with excessive maltodextrin. At the beginning of the culture each carbon source was added at the concentration of $0.5 \%(\mathrm{w} / \mathrm{v})$ and additional $1 \%(\mathrm{w} / \mathrm{v})$ was added at the $6 \mathrm{~h}$ of the cultivation (marked with an arrow).
Otherwise, related enzymes such as MalP and/or MalQ might have activity or substrate preference different from those of $E$. coli. Previous studies on glycogen metabolism in $E$. coli revealed that both maltose and maltodextrin contributed more to the glycogen accumulation than glucose by inducing the maltose utilization system [20]. On the flip side, glucose might turn off the maltose system via catabolic repression. Based on the results obtained in this study, $V$. vulnificus seemed to prefer maltodextrin to glucose or maltose in synthesizing glycogen, suggesting that synthesis of glycogen might be closely related to the maltodextrin metabolism. Furthermore, the results correlated well with those obtained by the agar plate assays (Fig. 1). In E. coli, maltose and maltodextin metabolisms are known to be mediated by the malPQ operon [20]. Since there was no gene annotated as $g \lg P$ in the genome of $V$. vulnificus, MalP might function as a phosphorylase for maltose, maltodextrin, and glycogen in the bacterium. Presence of a single phosphorylase might be responsible for accumulation of more glycogen than other bacteria by limiting utilization of the molecule in $V$. vulnificus.

\section{Effects of the malP and malQ genes on glycogen accumu- lation}

Since maltodextrin seemed to be a very important carbon source causing accumulation of glycogen to the highest level in $V$. vulnificus under the conditions examined in this study, effect of the genes related to the metabolism of maltodextrin was investigated using the malP and malQ mutants. Previously, malP and malQ were reported to be essential for the growth of $V$. vulnificus with maltose as a sole carbon source [17]. However, no information on the function of malP or malQ regarding maltodextrin and glycogen metabolism in $V$. vulnificus has been documented.

Accumulation of glycogen in the $V$. vulnificus mutants, malP $P^{-}$and malQ $^{-}$, was analyzed quantitatively and compared with that in WT by culturing in HI complex medium or M9 minimal medium for $14 \mathrm{~h}$ at $37^{\circ} \mathrm{C}$, using glucose or maltodextrin as an excessive carbon source. The two mutants accumulated much less amount of glycogen than the WT strain in HI medium (10.4-15.0\%) and almost none in M9 minimal medium (3.1-4.0\%) when the cultures were supplemented with $1 \%$ (w/v) maltodextrin (Fig. 5). In the M9 minimal medium, the 

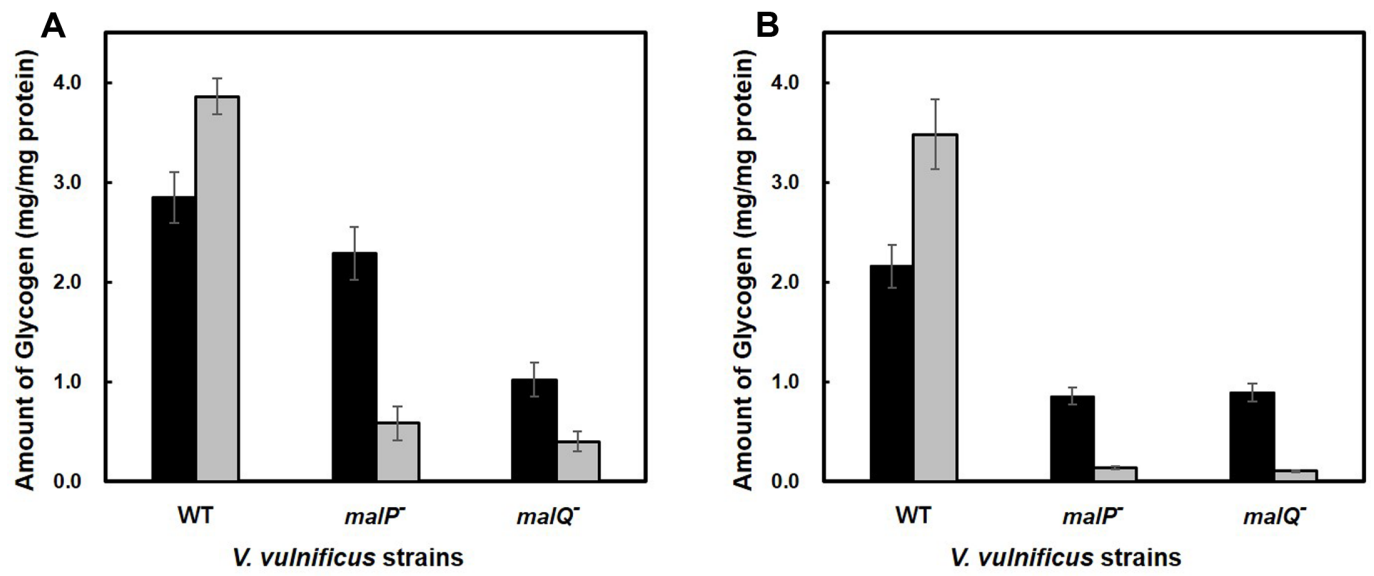

Fig. 5. Glycogen accumulation of the $V$. vulnificus strains in HI complex medium (A) and M9 minimal medium (B). The black columns represent the amount of glycogen from the medium containing additional glucose $(1 \% ; \mathrm{w} / \mathrm{v})$ as a carbon source and the gray columns additional maltodextrin $(1 \% ; \mathrm{w} / \mathrm{v})$. Glycogen samples were harvested at the $9 \mathrm{~h}$ after addition of each carbon source. WT, wild type; malP ${ }^{-}$, the knock-out mutant of malP; malQ ${ }^{-}$, the knock-out mutant of malQ.

mutants showed very poor growth since they could not use the carbon source efficiently. The malQ mutant affected glycogen accumulation slightly more than the malP mutant in both media, implying that glycogen was probably utilized by MalP that might function as GlgP, a glycogen phosphorylase, in V. vulnificus. This suggested that the malP and malQ genes probably be involved in the utilization of maltodextrin, which was closely related to glycogen metabolism in $V$. vulnificus. Previously, inactivation of the malQ gene was reported to inhibit secretion of cholera toxin and to reduce production of the mannose-sensitive hemagglutinin and the soluble hamagglutinin-protease in V. cholera [27]. This indicated that glycogen metabolism might have inter-relationship with other regulatory systems in bacteria.

Glycogen accumulation in $V$. vulnificus was also impaired in both media supplemented with $1 \%$ (w/v) glucose, but not as significant as those in the media supplemented with maltodextrin (Fig. 5), suggesting that the genes are somehow involved in glycogen accumulation caused by excessive glucose, too. Again, maltodextrin was the more effective carbon source than glucose for glycogen accumulation in the bacteria in all the cases. This could be due to glucose directly linked to glycolysis for energy generation and exerting catabolite repression on the maltodextrin metabolism. In the presence of excessive glucose, glycogen is synthesized via only the glucose dependent pathway, while both glucose dependent and maltodextrin dependent pathways contribute to glycogen accumulation in the presence of excessive maltodextrin. Therefore, maltodextrin degradation and disproportionation might be a more efficient way to increase glycogen in the bacterium. However, these results on glycogen metabolism in $V$. vulnificus was totally in opposite with those in $E$. coli, which accumulated more glycogen in the malP mutant with excessive glucose, maltose, and maltodextrin than WT; in the malQ mutant, more glycogen accumulated with glucose and maltodextrin but none with maltose [20]. In E. coli, the malQ mutant could not grow on maltose but could grow on longer maltodextrins such as maltoheptaose due to the action of MalP releasing glucose-1-P, while $E$. coli lacking MalP could grow on maltose and short maltodextrins [28]. Therefore, these genes seemed to be related to maltose and maltodextrin utilization of $E$. coli in a coordinative manner [21].

Based on the results, $V$. vulnificus seemed to have multiple routes for the synthesis and utilization of glycogen, which are unique to the pathogen. Especially, utilization of maltodextrin by MalP and MalQ in $V$. vulnificus might be related to accumulation of glycogen more closely than in $E$. coli. When compared with WT, malP $P^{-}$and malQ $^{-}$could accumulate much less glycogen when glucose was added as an excessive carbon source. The malP and malQ mutants could not utilize glucose appropriately for glycogen synthesis even though glucose generally could be used easily than longer malto dextrins. 


\section{Structure of glycogen accumulated in $\mathbf{V}$. vulnificus}

Utilization of stored glycogen is very closely related to the survival of bacteria under limiting growth conditions and greatly dependent on the structure of the molecule. Glycogen molecules take globular shape by forming branches of maltodextrins via $\alpha$-1,6-glycosidic linkage. Bacterial glycogen molecule may have 12 tiers of side chain branches with average chain lengths of 7-12 glucose units [15]. Glycogen with short average chain length has been known to be degraded very slowly and lead to longer survival under starvation conditions [12]. The structure was likely to be dynamically modulated according to the ever changing intra-cellular energy flux and availability of nutrients.
As an effort to elucidate the structural characteristics of glycogen synthesized by $V$. vulnificus, side chain length distribution of the molecules in the WT, malPand $\mathrm{malQ}^{-}$strains was analyzed under various culture conditions (Fig. 6 and 7). The glycogen samples were treated with isoamylase before they were subjected to HPAEC. First, change in the side chain length of glycogen was monitored during the growth of the WT strain using LB broth supplemented with $1 \%$ (w/v) maltodextrin at the $6 \mathrm{~h}$ of culture. No side chain released from the core chain by isoamylase was detected in glycogen extracted from the cells before the addition of maltodextrin (Fig. 6C). However, side chains with various lengths began to be detected in $2 \mathrm{~h}$ of maltodextrin supplementa-
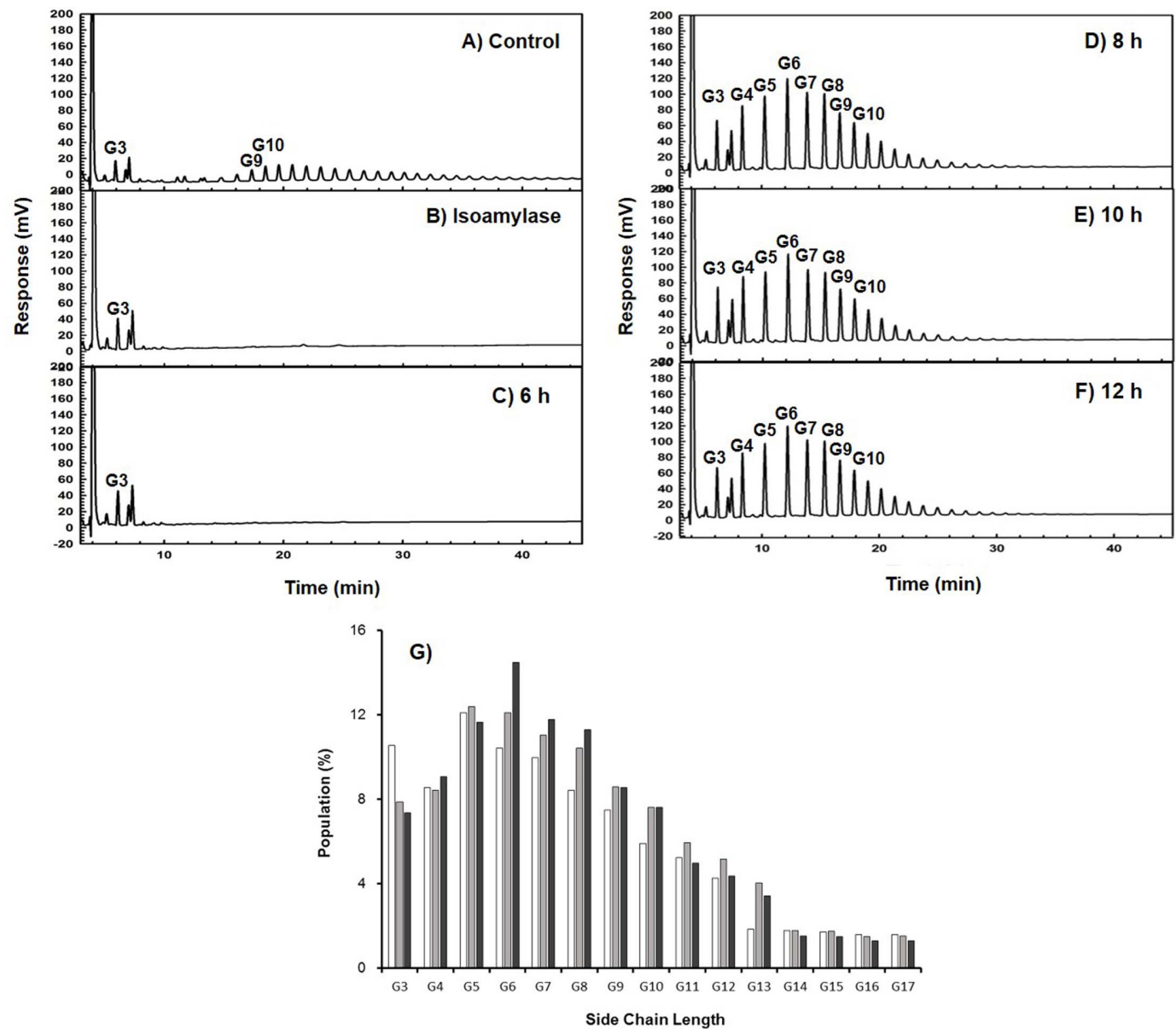

Fig. 6. Side chain length distribution of glycogen from wild type $\mathbf{V}$. vulnificus. The cells were cultured in LB broth containing $2 \%(\mathrm{w} / \mathrm{v}) \mathrm{NaCl}$ and supplemented with $1 \%(\mathrm{w} / \mathrm{v})$ maltodextrin at the $6 \mathrm{~h}$ of cultivation. Aliquots of the culture were taken at every $2 \mathrm{~h}$ to extract glycogen and the glycogen samples were treated with isoamylase before subjected to HPAEC (C-F). Control was bovine glycogen treated with isoamylase (A), LB broth containing the enzyme was used as another negative control (B). Population of side chains in various lengths was analyzed according to culture time $(G)$. White bars represents those from the $8 \mathrm{~h}$ culture; grey bars, $10 \mathrm{~h}$; black bars, $12 \mathrm{~h}$. 
tion (Fig. 6D-F). The range of side chain length distribution was narrower and the lengths of major side chains were shorter than glycogen from $E$. coli and B. subtilis $[21,26]$. The side chains of G5-G8 were abundant with G6 becoming more abundant and the side chains growing longer as the culture time extended (Fig. 6G). The overall pattern for side chains longer than G13 was kept unchanged during the course of growth.

Glycogen was also extracted from the WT, malP-, and malQ $Q^{-}$cultures in HI or M9 minimal broth that was supplemented with $1 \%(\mathrm{w} / \mathrm{v})$ glucose or maltodextrin at the $6 \mathrm{~h}$ of cultivation and incubated further for $9 \mathrm{~h}$. The glycogen samples were treated with isoamylase and subjected to HPAEC analysis (Fig. 7). Glycogen samples from $V$. vulnificus WT cultured in the media supplemented with glucose had more side chains of G6, while those from the media with excessive maltodextrin had side chains of G4 and G5 slightly more abundant than G6. These data implied that glycogen structure might be modulated according to available carbon source. As mentioned in the above analysis, the mutants not only accumulated less glycogen than wild type but also the side chains released from the molecules were very limited. Especially, glycogen extracted from the alP $^{-}$and malQ ${ }^{-}$cultures supplemented with maltodextrin did not exhibited detectable side chains under the conditions for the analysis. On the other hand, glycogen molecules from the mutants showed side chain length distribution similar to those of wild type, when they were extracted from the cultures supplemented with excessive glucose. These data suggested that malP and malQ were indeed involved in glycogen synthesis and modulation of the structure when the bacterium faced excessive maltodextrin in the cell. The malQ mutant accumulated much less glycogen than WT in M9 minimal medium supplemented with glucose or in HI broth supplemented with mlatodextrin, but accumulated glycogen in amounts similar to those of malP $^{-}$under the conditions. However, the two glycogen samples extracted from $\mathrm{malQ}^{-}$released very little side chains, which might indicate that the molecules did not have much side chains. Therefore, MalQ might affect both pathways of glycogen synthesis and be responsible for disproportionation reactions to provide dextrins to form side chains.

Glycogen side chain length of $E$. coli has been reported to be longer than that of $V$. vulnificus with major chain length of G12 when the bacteria was cultured in broth supplemented with maltodextrin as a carbon source [21]. The range of side chains was much wider in E. coli (G3G25) than $V$. vulnificus (G4 <G20). In B. subtilis, the

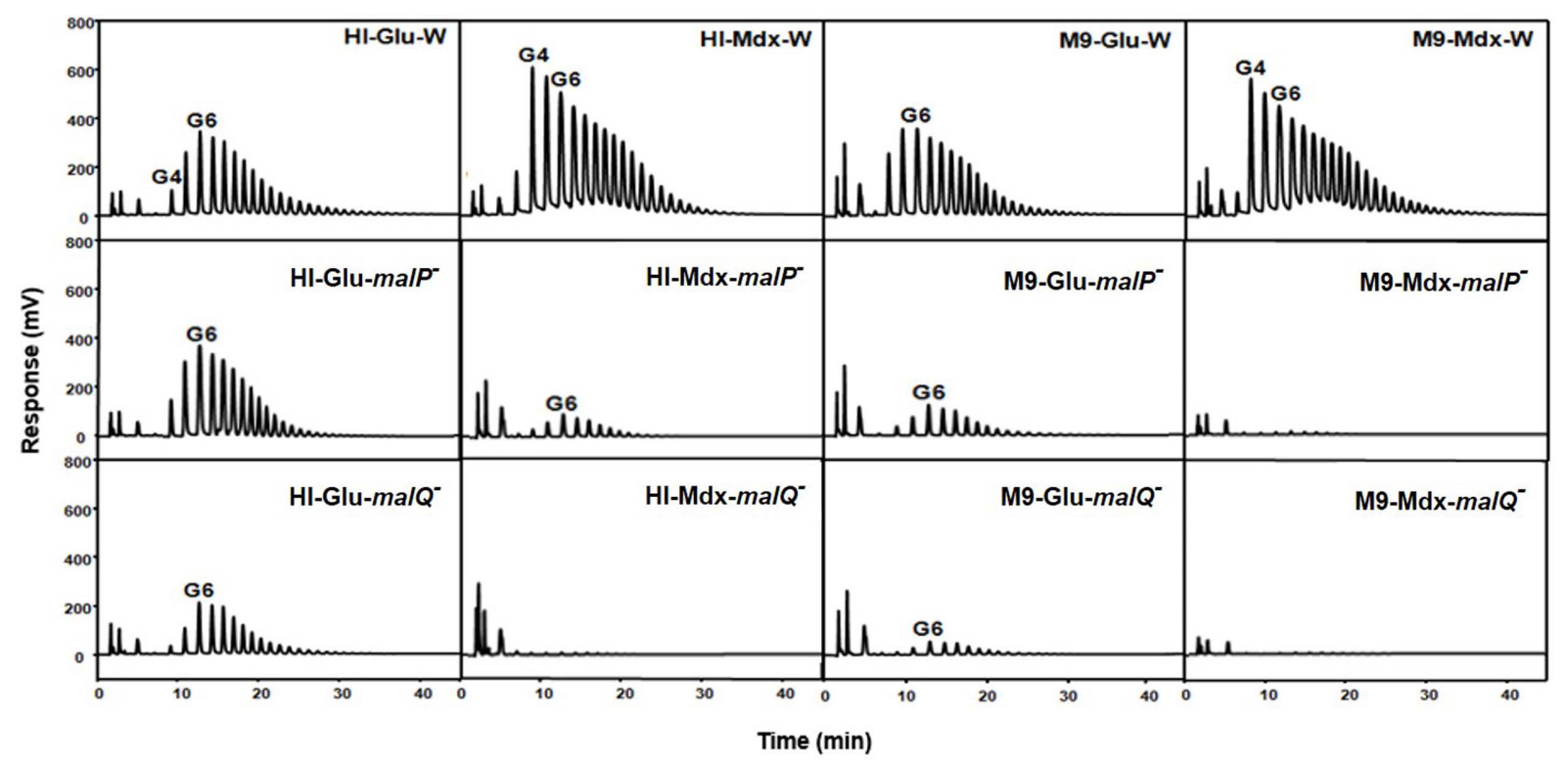

Fig. 7. Side chain length distribution of glycogen from various $\mathbf{V}$. vulnificus strains. The wild type (W), malP $P^{-}$and malQ $^{-}$strains were cultured in $\mathrm{HI}$ or $\mathrm{M} 9$ minimal broth supplemented with additional $1 \%(\mathrm{w} / \mathrm{v})$ glucose (Glu) or maltodextrin (Mdx) at the $6 \mathrm{~h}$ and cultured further for $9 \mathrm{~h}$ before collected for glycogen extraction. The glycogen samples were treated with isoamylase before they were subjected to HPAEC. 
pattern of side chain length distribution of glycogen was similar to that of $V$. vulnificus in that G4 or G6 being most abundant [26]. However, side chain length distribution of glycogen form B. subtilis (G3 > G30) was wider than that of $V$. vulnificus. However, glycogen in B. subtilis could be detected only when the glg operon was overexpressed in the cell [26]. Glycogen reserve in B. subtilis was reported that they were used as the energy source for sporulation of Bacilli, a terminal decision to survive [29]. The pattern of side chain length distribution was observed to be maintained in $V$. vulnificus as time passed and more glycogen was accumulated (between 8 and 12 h; Fig. 6D-E). However, the structure of glycogen in $B$. subtilis began to be changed due to degradation of glycogen in cultures older than $10 \mathrm{~h}$ [26]. These results implied that $V$. vulnificus would have good chance to survive under starvation conditions (probably outside the hosts), utilizing glycogen reserve as the carbon and energy source. This is supported by previous reports that bacteria having highly branched glycogen with short chain lengths (G7-G9) would degrade it more slowly than those having glycogen with longer chain lengths (G12-15) [30-32]. Recent studies on the relationship between the structure of glycogen and bacterial durability also have revealed that ACL of glycogen could affect the degradation rate of glycogen [12]. Bacteria that synthesized glycogen with short ACL had more $\alpha-1,6$-glycosidic linkages, preventing glycogen from being degraded by glycogen debranching enzymes. $V$. vulnificus was also unique in its glycogen branching enzyme that transferred short side chains more efficiently than other bacterial glycogen branching enzymes and led to accumulation of highly branched glycogen with short side chains [33].

Based on all the results obtained in this study, we propose that $V$. vulnificus might operate at least more than two pathways for the synthesis and degradation of glycogen depending on the following factors; nutritional conditions (i.e., carbon sources) during the growth and habitats (i.e., host or aquatic environment). Also, these might be coordinated with the structure of glycogen highly branched with short side chains, which was modulated by the unique genetic constitution and enzyme activity involved in the glycogen metabolism. A previous report on $V$. cholera showed that pathogenic strain accumulated more glycogen than non-pathogenic strains and had better chance to transmit successfully in a variety of environmental niches [8]. This was supported by a study on pathogenic $E$. coli, which reported that glycogen metabolism was significant for colonization in the murine intestine [34]. All these implied that glycogen metabolism might be regulated in coordinative modes with various response systems to survive and transmit in nature such as pathogenesis. In order to elucidate unique glycogen metabolism and its regulation in $\mathrm{V}$. vulnificus, further studies on the genes involved glycogen metabolism need to be carried out and they would provide a good model for the role of glycogen in transmission and pathogenicity of bacteria.

\section{Acknowledgments}

We gratefully acknowledge excellent graphical and technical support for the manuscript by Miss So-Rah Park. This study was supported by a grant from Incheon National University in 2014.

\section{Conflict of Interest}

The authors have no financial conflicts of interest to declare.

\section{References}

1. Tison DL, Kelly MT. 1986. Virulence of Vibrio vulnificus strains from marine environments. Appl. Environ. Microbiol. 51: 1004-1006.

2. Chen CY, Wu KM, Chang YC, Chang CH, Tsai HC, Liao TL, et al. 2003. Comparative genome analysis of Vibrio vulnificus, a marine pathogen. Genome Res. 13: 2577-2587.

3. Wallington J, Ning J, Titheradge MA. 2008. The control of hepatic glycogen metabolism in an in vitro model of sepsis. Mol. Cell. Biochem. 308: 183-192.

4. Park SD, Shon HS, Joh NJ. 1991. Vibrio vulnificus septicemia in Korea: clinical and epidemiologic findings in seventy patients. J. Am. Acad. Dermatol. 24: 397-403.

5. Schild S, Tamayo R, Nelson EJ, Qadri F, Calderwood SB, Camilli A. 2007. Genes induced late in infection increase fitness of Vibrio cholerae after release into the environment. Cell Host Microbe. 2: 264-277.

6. Jones MK, Oliver JD. 2009. Vibrio vulnificus: Disease and Pathogenesis. Infect. Immun. 77: 1723-1733.

7. Nelson EJ, Harris JB, Morris JG, Calderwood Jr SB, Camilli A. 2009. Cholera transmission: the host, pathogen and bacteriophage dynamic. Nature 7: 693-702.

8. Bourassa L, Camilli A. 2009. Glycogen contributes to the environmental persistence and transmission of Vibrio cholerae. Mol. Microbiol. 72: 124-138.

9. Iglesias AA, Preiss J. 1992. Bacterial glycogen and plant starch 
biosynthesis. Biochem. Edu. 20: 196-203.

10. Preiss J, Romeo T. 1989. Physiology, biochemistry and genetics of bacterial glycogen synthesis. Adv. Bacter. Physiol. 30: 184-238.

11. Wang L, Wise MJ. 2011. Glycogen with short average chain length enhances bacterial durability. Naturwissenschaften 98: 719-729.

12. Preiss J. 2009. Glycogen: biosynthesis and regulation. EcoSal Plus doi: 10.1128/ecosalplus.4.7.4.

13. Montero M, Almagro G, Eydallin G, Viale AM, Muñoz FJ, Bahaji $A$, et al. 2011. Escherichia coli glycogen genes are organized in a single glgBXCAP transcriptional unit possessing an alternative suboperonic promoter within $g l g C$ that directs $g l g A P$ expression. Biochem. J. 433: 107-117.

14. Lombardo M, Michalski J, Martinez-Wilson H, Morin C, Hilton T, Osorio CG, et al. 2007. An in vivo expression technology screen for Vibrio cholerae genes expressed in human volunteers. Pro. Nat. Acad. Sci. 104: 18229-18234.

15. Park K. 2015. Roles of enzymes in glycogen metabolism and degradation in Escherichia coli. J. Appl. Glycosci. 62: 37-45.

16. Pugsley AP, Dubreuil C. 1988. Molecular characterization of malQ, the structural gene for the Escherichia coli enzyme amylomaltase. Mol. Microbiol. 2: 473-479.

17. Lim MS, Lee MH, Lee JH, Ju H, Park NY, Jeong HS, et al. 2005. Identification and characterization of the Vibrio vulnificus malPQ operon. J. Microbiol. Biotechnol. 15: 616-625.

18. Damotte M, Cattaneo J, Sigal N, Puig J. 1968. Mutants of Escherichia coli $\mathrm{K} 12$ altered in their ability to store glycogen, Biochem. Biophys. Res. Comm. 35: 916-920.

19. Dauvilleé D, Kinderf IS, Li Z, Kosar-Hashemi B, Samuel MS, Rampling $\mathrm{L}$, et al. 2005. Role of the Escherichia coli glgX gene in glycogen metabolism. J. Bacteriol. 187: 1465-1473.

20. Park J, Shim J, Tran PL, Hong I, Yong H, Oktavina EF, et al. 2011. Role of maltose enzymes in glycogen synthesis by Escherichia coli. J. Bacteriol. 193: 2517-2526.

21. Scott Jr. TA, Melvin EH. 1953. Determination of dextran with anthrone. Anal. Chem. 25: 1656-1661.

22. Belanger AE, Hatfull GF. 1999. Exponential-phase glycogen recycling is essential for growth of Mycobacterium smegmatis. J. Bacteriol. 181: 6670-6678.

23. Paalman JW, Verwaal R, Slofstra SH, Verkleij AJ, Boonstra J, Verrips
CT. 2003. Trehalose and glycogen accumulation is related to the duration of the G1 phase of Saccharomyces cerevisiae. FEMS Yeast Res. 3: 261-268.

24. Holme T, Palmstierna H. 1956. Changes in glycogen and nitrogen-containing compounds in Escherichia coli B during growth in deficient media. I. Nitrogen and carbon starvation. Acta Chem. Scand. 10: 578-586.

25. Preiss J. 1984. Bacterial glycogen synthesis and its regulation. Annu. Rev. Microbiol. 38: 419-458.

26. Shim J, Park J, Hong J, Kim KW, Kim M, Auh J, et al. 2009. Role of maltogenic amylase and pullulanase in maltodextrin and glycogen metabolism of Bacillus subtilis 168. J. Bacteriol. 191: 48354844.

27. Lang H, Jonson G, Holmgren J, Palva ET. 1994. The maltose reguIon of Vibrio cholerae affects production and secretion of virulence factors. Infect. Immun. 62: 4781-4788.

28. Palmer TN, Wober G, Whelan WJ. 1973. The pathway of exogenous and endogenous carbohydrate utilization in Escherichia coli: a dual function for the enzymes of the maltose operon. Eur. J. Biochem. 39: 601-612.

29. Slock JA, Stahly DP. 1974. Polysaccharide that may serve as a carbon and energy storage compound for sporulation in Bacillus cereus. J. Bacteriol. 12: 399-406.

30. Zevenhuizen LP, Ebbink AG. 1974. Interrelations between glycogen, poly-beta-hydroxybutyric acid and lipids during accumulation and subsequent utilization in a Pseudomonas. Antonie Van Leeuwenhoek 40: 103-120.

31. Strange RE. 1968. Bacterial glycogen and survival. Nature 220: 606-607.

32. Boylen CW, Mulks MH. 1978. Survival of Coryneform bacteria during periods of prolonged nutrient starvation. J. Gen. Microbiol. 105: 323-354.

33. Jo H, Park S, Jeong H, Kim J, Park J. 2015. Vibrio vulnificus glycogen branching enzyme preferentially transfers very short chains: $\mathrm{N} 1$ domain determined the chain length transferred. FEBS Lett. 589: 1089-1094.

34. Jones SA, Jorgensen M, Chowdhury FZ, Rodgers R, Hartline J, Leatham MP, et al. 2008. Glycogen and maltose utilization by Escherichia coli 0157: H7 in the mouse intestine. Infect. Immun. 76: 2531-2540. 